Aim of the study: An ERAS protocol provides the latest perioperative care principles, whose primary aim is to reduce complication rates, and therefore mortality. The aim of this study is to establish the progress of the ERAS pathway implementation in our gynaecology department.

Material and methods: This was a retrospective analysis of two sets of 100 consecutive medical records: patients treated before (PRE-ERAS) and after (ERAS) introduction of the ERAS protocol. All patients were comparable and all underwent major gynaecological surgery. Patients as well as medical and nursing staff were informed about the proposed preparation, surgical management and postoperative routine.

Results and conclusions: Patients were given supper and drank water during the night. Laparoscopic surgery was used in $44 \%$ and spinal anaesthesia was given for open surgery in 43 study patients. Use of drains was reduced only by $23 \%$, bowel preparation by $15 \%$. Intravenous fluid administration was reduced by $22 \%$. Use of postoperative morphine was minimised to 12 patients. Postoperative nausea was managed with the regular use of anti-emetics. Anti-coagulation was given to $80 \%$ of the study group. Difficulties in the introduction of the ERAS protocol were due to refusal by some patients to mobilise and eat early postoperatively. Patients in the ERAS programme group were discharged earlier.

Further information about the ERAS protocol in the media would facilitate patients' education among conservative society. In order to introduce new and innovative treatment methods, one has to take into account the cultural and ideological factors, especially when patient involvement is essential.

Key words: ERAS, perioperative care.

Contemp Oncol (Pozn) 2017; 21 (3): 240-243 DOI: https://doi.org/10.5114/wo.2017.69589

\section{Implementation of an enhanced recovery after surgery (ERAS) protocol in a gynaecology department - the follow-up at 1 year}

\author{
Tomasz Nikodemski ${ }^{1}$, Agnieszka Biskup ${ }^{2}$, Aleksandra Taszarek², \\ Małgorzata Albin ${ }^{3}$, Anita Chudecka-Głaz ${ }^{2}$, Aneta Cymbaluk-Płoska ${ }^{1}$, \\ Janusz Menkiszak ${ }^{1}$
}

${ }^{1}$ Health Care Centre of the Ministry of Interior and Administration, Szczecin, Poland ${ }^{2}$ Department of Gynecological Surgery and Gynecological Oncology of Adults and Adolescents, Pomeranian Medical University, Szczecin, Poland

${ }^{3}$ The Lister Hospital, Chelsea Bridge Road, London SW1W 8RH, United Kingdom

\section{Introduction}

The introduction of the ERAS (Enhanced Recovery After Surgery) programme is becoming standard implementation of surgical care in the hospital environment. The historical significance of ERAS has been established since 1990 (REF) in general surgery, orthopaedics and urology [1]. ERAS resulted in increased patient satisfaction, shorter hospital stays, reduced postoperative complications and reduced treatment costs, which contribute to increased hospital productivity. The aim of this study is to introduce a number of ERAS recommendations into the management of gynaecological procedures in our institution.

There are a number of reports in the literature on the implementation of the ERAS programme into the care of gynaecological patients [2-4]. Minimally invasive surgery is advisable in order to reduce surgical complications, wound infections, incidence of deep vein thrombosis and hospital stay [5]. There is a strong opinion that the majority of gynaecological procedures can be done without mechanical bowel preparation (enema) [6]. One of the demonstrated advantages of restricting the administration of intravenous fluids to surgical patients is the reduction of pulmonary complications [7]. Another recommendation is the avoidance of opioids, which can cause nausea, vomiting and ileus [8].

\section{Material and methods}

Groups of patients

The study compared two groups of women undergoing hysterectomy. Both groups had similar indications for surgery, with the majority being oncology patients. This study took place between July 2014 and June 2015, with 100 patients included in both the study and control groups. The control group was assessed from July to December 2014 and had data extracted from patients' medical records. The study group was assessed from January to June 2015, when the ERAS pathway was implemented. This permitted the primary aim of the study: to evaluate whether there was a significant difference in the length of hospital stay.

\section{Preoperative interview}

All 100 patients were interviewed preoperatively and pre-assessed. During the interview, the women were given detailed information about 
the proposed management including the reduced fasting time, drinking water preoperatively, avoidance of premedication, importance of early mobilisation after surgery and anticoagulation. They were also informed about the planned method of surgery, anaesthesia and the choice of postoperative pain relief. All of the patients were given a chance to discuss the proposed plan and were reassured about its benefits.

\section{Liaison with gynaecologists}

Discussions and presentations to the gynaecology team were provided prior to the commencement of the study, in order to agree on the principles of the ERAS protocol.

Topics included the importance of the introduction of minimally invasive surgery and reduction of the use of drains and urinary catheters or their early removal. In line with the ERAS recommendations, mechanical bowel preparation should only be used if specifically indicated.

\section{Anaesthetic technique}

Premedication with a sedative was avoided unless the patient insisted on receiving one. The number of laparoscopic procedures in this study group increased, which led to a proportional increase in general anaesthesia, as this was the method of choice for laparoscopic surgery. For open surgery, general anaesthesia or spinal anaesthesia was used, with the option of added intrathecal morphine.

The patient's body temperature was recorded throughout surgery and active warming included a hot air blanket, warming mattress and fluid warmer, which were used routinely. Intravenous crystalloids (Ringers lactate solution) were given to maintain a stable balance without under- or overloading the patient. All patients received a prophylactic dose of intravenous antibiotic (cefuroxime) and a subcutaneous dose of anticoagulant (nadroparin calcium).

\section{Analgesia}

All patients were prescribed a pre-emptive dose of analgesia prior to the surgery in the form of ketoprofen, metamizole sodium and diclofenac sodium.

Opiate medications were avoided in the postoperative period and non-opiate oral drugs were used instead whenever possible.

Postoperative regular analgesia was provided with paracetamol, metamizole sodium, diclofenac sodium and oxycodone, with subcutaneous morphine sulphate for

Table 1. Patients' characteristics

\begin{tabular}{|lcc|}
\hline Parameter & PRE-ERAS & ERAS \\
\hline Age & 54.83 & 51.99 \\
BMI & 28.01 & 26.7 \\
Hypertension & $36 \%$ & $35 \%$ \\
\hline Diabetes & $14 \%$ & $10 \%$ \\
\hline Chronic renal disease & $3 \%$ & $1 \%$ \\
\hline Ischemic heart disease & $3 \%$ & $4 \%$ \\
\hline COPD & $2 \%$ & 0 \\
\hline
\end{tabular}

breakthrough pain. Anti-emetics were given on a regular basis in the form of ondansetron hydrochloride and dexamethasone.

\section{Antithrombotic prophylaxis}

This was done with regular administration of nadroparin calcium.

\section{Diet}

Patients were allowed to have supper in the evening before surgery and were allowed to eat "at will" postoperatively on the day of surgery or the first postoperative day if preferred.

\section{Mobilisation}

Patients were advised to mobilise as early as possible on the operative day or at least on the first postoperative day.

\section{Statistics}

Statistical analysis was performed using commercial software (Statistica, version 5.0, StatSoft, Krakow, Poland). Numerical variables were compared using Fisher's exact test. Categorical variables with non-normal distributions were compared by means of the Mann-Whitney U-test. Differences with $p$-values $<0.05$ were defined as significant.

\section{Results}

Both groups were similar regarding their demographics. Patients' age, BMI and incidence of medical conditions such as hypertension, diabetes, chronic renal disease, ischemic heart disease and COPD did not differ significantly between the two groups (Table 1).

Before the introduction of the ERAS programme, patients had their last meal at 1 p.m. on the eve of the operation and clear liquids were allowed until 10 p.m. In July 2014, patients' preoperative nutrition practices changed. From then, on the day before surgery, supper was the last meal, and patients were permitted to drink water during the night.

ERAS programme elements introduced in our institution and its implementation rate are presented in Table 2.

All study group patients were interviewed and full comprehensive information was given to them regarding the management of the forthcoming procedure.

The mechanical bowel preparation in the form of an enema was abandoned in 15\% of the study group as compared to $3 \%$ of the control group ( $p=0.007)$.

The administration of premedication was reduced in the study group, with $30 \%$ of women not requesting it as compared to only $5 \%$ in the control group $(p<0.0001)$. In line with the introduction of restrictive intravenous fluid therapy, $22 \%$ of patients in the study group compared with $2 \%$ in the control group received up to $500 \mathrm{ml}$ of crystalloids perioperatively $(p<0.0001)$.

In the study group 43 patients were given spinal anaesthesia, and out of these 14 also received spinal morphine. Administration of spinal opiates is not a routine practice 
Table 2. Effectiveness of implementation of the ERAS programme

\begin{tabular}{|c|c|c|c|c|}
\hline ERAS protocol goals & Pre-ERAS & ERAS & $\begin{array}{l}\text { Statistical } \\
\text { significance }\end{array}$ & $\begin{array}{l}\text { Efficiency of } \\
\text { implementation }\end{array}$ \\
\hline No bowel prep & $3 \%$ & $15 \%$ & $p=0.007$ & poor \\
\hline No pre-med & $5 \%$ & $30 \%$ & $p<0.0001$ & poor \\
\hline Restrictive i.v. fluid regime & $2 \%$ & $22 \%$ & $p<0.0001$ & poor \\
\hline Laparoscopic surgery & $12 \%$ & $44 \%$ & $p<0.0001$ & satisfactory \\
\hline No drains & $0 \%$ & $23 \%$ & $p<0.0001$ & poor \\
\hline Non-opioid oral analgesic & $20 \%$ & $88 \%$ & $p<0.0001$ & good \\
\hline $\begin{array}{l}\text { Prevention of post-operative nausea and } \\
\text { vomiting }\end{array}$ & $56 \%$ & $86 \%$ & $p<0.0001$ & good \\
\hline Routine mobilisation at the day of surgery & $0 \%$ & $45 \%$ & $p<0.0001$ & satisfactory \\
\hline Early removal of catheters (in 24 h) & $7 \%$ & $70 \%$ & $p<0.0001$ & good \\
\hline \multirow[t]{2}{*}{ Oral nutrition } & $\begin{array}{l}\text { the day of surgery } \\
\qquad-1 \%\end{array}$ & $\begin{array}{l}\text { the day of surgery } \\
\qquad-13 \%\end{array}$ & $p=0.002$ & good \\
\hline & $\begin{array}{l}\text { first postoperative } \\
\text { day }-8 \%\end{array}$ & $\begin{array}{l}\text { first postoperative } \\
\text { day }-79 \%\end{array}$ & $p<0.0001$ & \\
\hline
\end{tabular}

in Poland. All patients received oxygen throughout the surgery. The remaining 57 patients in the study group received general anaesthesia, with addition of intravenous morphine required in 10 women during surgery. In the control group 64 patients received spinal anaesthesia, 36 of these with the addition of spinal morphine. The remaining 36 patients in the control group received general anaesthesia with similar drugs as used in the study group, and intravenous morphine was required in 22 women.

The surgical technique was successfully changed to laparoscopy from $12 \%$ in the control group to $44 \%$ in the study group $(p<0.0001)$. The usage of surgical drains was also altered, as they were not inserted in $23 \%$ of the study group patients, whereas all 100 patients in the control group had drains inserted $(p<0.0001)$.

Patients in the study group received anti-emetic prophylaxis with regular ondansetron hydrochloride and dexamethasone at induction of anaesthesia in $86 \%$ as compared to $56 \%$ of the control group ( $p<0.0001$ ).

After commencing the ERAS programme, post-operative nausea and vomiting requiring pharmacological intervention occurred less frequently: $6 \%$ in the study group compared to $23 \%$ in the control group ( $p=0.001$ ).

Postoperative analgesia in both groups was managed with regular medications - metamizole sodium, ketoprofen, and diclofenac sodium. One dose of oxycodone $5 \mathrm{mg}$ was given to 4 patients. Administration of additional morphine sulphate postoperatively was significantly reduced in the study group (12 patients) as compared to the control group (80 patients) $(p<0.0001)$.

For antithrombotic prophylaxis 80 patients in the study group and 64 in the control group received nadroparin calcium routinely. No thrombotic complications were observed in either group.

Post-operative early mobilisation on the day of surgery was achieved in $45 \%$ of the study group patients. On the other hand, none of the control group patients mobilised on the day of the operation $(p<0.0001)$. In regards to eat- ing post-operatively, $13 \%$ of patients in the study group compared to $1 \%$ in the control group opted to eat the first meal on the day of surgery. This rate was increased on the first postoperative day, with $79 \%$ of the study group compared to $8 \%$ in the control group eating their first meal $(p<0.0001)$. Urinary catheters were removed within 24 hours after surgery in $70 \%$ of the study group patients and in $7 \%$ of the control group patients ( $p<0.0001)$.

The length of hospital stay in the ERAS study group was shorter, with a median of 6 , compared to 7 days in the preERAS control group $(p=0.0001)$. Patients from the pre-ERAS control group left the hospital on average on the fifth postoperative day and in the ERAS study group on the fourth day ( $p=0.0001)$.

Complications after surgery in the form of postoperative nausea and vomiting, requirement for blood transfusion, wound resuturing and wound infection occurred more often in patients operated on before the introduction of the ERAS programme, but this difference was not statistically significant (23 vs. 17; $p=0.06$ ).

\section{Discussion}

There is evidence in the literature showing that ERAS is beneficial in the management of patients by improving the quality of care and use of resources. Reduced hospital stay and improved patient quality of life are achieved after the introduction of the programme. An important fact to highlight is that ERAS does not require additional technology and equipment. The introduction of the programme necessitated the cooperation and coordination between all teams involved and the willingness of patients.

We encountered a number of difficulties in following the proposed programme: a number of patients were reluctant to avoid premedication, some objected to early mobilisation, and not everyone was keen to eat early postoperatively. It is also fundamental to convince staff to move on from the traditional approach of surgical patient 
management [9]. This would require thorough preparation and training of staff to be compliant with the new standards.

Efficiency and benefits of using the ERAS programme depend primarily on the commitment and expertise of the staff. It is assumed that the whole process should be guided by the philosophy of patient-oriented nursing. It would seem that this programme increases the workload for nurses by giving additional information to patients in the preoperative period and motivating the patient to mobilise or eat early after surgery.

The patient managed according to the ERAS protocol becomes independent earlier and does not require oneon-one nursing care. In addition, fewer postoperative complications and the possibility of earlier patient discharge reduce nursing input, with reduced costs incurred by the clinic. The potential benefits of implementation of ERAS include greater patient satisfaction due to early rehabilitation, reducing discomfort, and shorter hospital stay and cost per treatment [10].

On the other hand, the total costs are rising, because at the same time there is an increase in the number of treated patients. Handling a smaller number of patients does not generate major financial savings due to the fixed department costs. The introduction of the ERAS can increase the efficiency and productivity of the unit, allowing better use of resources.

To conclude, the ERAS protocol is a modern interdisciplinary programme of perioperative patient care, increasing safety and comfort, while shortening the time of hospitalisation and lowering the cost of treatment. Implementation of the ERAS programme increases patient satisfaction with treatment, reduces the number of postoperative complications, shortens hospital stay and reduces treatment costs, which contributes to increased overall hospital productivity. This programme consists of the introduction of a package of measures, which had to be combined together to give the best therapeutic effect. Therefore, the ERAS protocol should be implemented for all gynaecological patients.

The authors declare no conflict of interest.

\section{References}

1. Kehlet H, Dahl JB. Anaesthesia, surgery, and challenges in postoperative recovery. Lancet 2003; 362: 1921-8.

2. Nelson G, Kalogera E, Dowdy SC. Enhanced recovery pathways in gynecologic oncology. Gynecologic Oncology 2014; 135: 586-94.

3. Wijk L, Franzen K, Ljungqvist O, Nilsson K. Implementing a structured Enhanced Recovery After Surgery (ERAS) protocol reduces length of stay after abdominal hysterectomy. Acta Obstet Gynecol Scand 2014; 93: 749-56.

4. Kalogera E, Bakkum-Gamez JN, Jankowski CJ, et al. Enhanced recovery in gynecologic surgery. Obstet Gynecol 2013; 122 (2 Pt 1): 319-28.

5. Kluivers KB, Cate Ten FA, Bongers MY, et al. Total laparoscopic hysterectomy versus total abdominal hysterectomy with bilateral salpingo-oophorectomy for endometrial carcinoma: a randomised controlled trial with 5-year follow-up. Gynecol Surg 2011; 8: 427-34.
6. Arnold A, Aitchison LP, Abbott J. Preoperative mechanical bowel preparation for abdominal, laparoscopic, and vaginal surgery: a systematic review. J Minim Invasive Gynecol 2015; 22: 737-52

7. Kehlet $\mathrm{H}$. Postoperative ileus - an update on preventive techniques. Nat Clin Pract Gastroenterol Hepatol 2008; 5: 552-8.

8. Misiołek H, Mayzner-Zawadzka E, Dobrogowski J, Wordliczek J. Zalecenia postępowania w bólu ostrym i pooperacyjnym. Bol 2011; 12: $9-33$

9. de Groot JJ, van Es LE, Maessen JM, Dejong CH, Kruitwagen RF, Slangen BF. Diffusion of enhanced recovery principles in gynecologic oncology surgery: is active implementation still necessary? Gynecologic Oncology 2014; 134: 570-5.

10. Yoong W, Sivashanmugarajan V, Relph S, et al. Can enhanced recovery pathways improve outcomes of vaginal hysterectomy? Cohort control study. J Minim Invasive Gynecol 2014; 21: 83-9.

\section{Address for correspondence}

\section{Tomasz Nikodemski}

Health Care Centre of the Ministry of Interior and Administration Jagielońska 44

70-382 Szczecin, Poland

e-mail: nikodemski@me.com

Submitted: 25.02 .2017

Accepted: $\quad 9.04 .2017$ 